\title{
Progression of Dental Caries and Tooth Loss between the Third and Fourth Decades of Life: A Birth Cohort Study
}

\author{
J.M. Broadbent ${ }^{a}$ W.M. Thomson ${ }^{a}$ R. Poulton ${ }^{b}$ \\ ${ }^{a}$ Department of Oral Sciences, Faculty of Dentistry and ${ }^{b}$ Department of Preventive and Social Medicine, \\ Dunedin School of Medicine, University of Otago, Dunedin, New Zealand
}

\section{Key Words}

Caries - Tooth loss

\begin{abstract}
The majority of what is known of the experience of dental caries among adults is from cross-sectional studies, and there are surprisingly few population-level longitudinal studies of dental caries among adults. Dental examinations were conducted at age 26 and again at age 32 among participants in a longstanding prospective study of a birth cohort born in Dunedin (New Zealand) in 1972/1973. Some 901 individuals (88.8\%) were dentally examined at both ages. The mean number of remaining teeth and tooth surfaces fell between 26 and 32 , reflecting ongoing tooth loss. The overall prevalence of caries rose from 94.9 to $96.8 \%$, while there were greater increases in the proportion with caries-associated tooth loss (from 10.8 to $22.8 \%$ ). Caries experience was greatest in the molar teeth and upper premolars, and was lowest in the lower anterior teeth. The mean crude caries increment (CCl) was 5.0 surfaces (SD 6.6); 681 (75.5\%) experienced $1+\mathrm{CCl}$, and the mean $\mathrm{CCl}$ among those individuals was 6.6 surfaces (SD 6.9). Substantial dental caries and tooth loss experience occur as people move from the third into the fourth decade of life.
\end{abstract}

Copyright ( 2006 S. Karger AG, Basel

\section{KARGER}

Fax +4161306 1234 E-Mail karger@karger.ch www.karger.com
(C) 2006 S. Karger AG, Basel

0008-6568/06/0406-0459\$23.50/0

Accessible online at: www.karger.com/cre
The majority of what is known of the experience of dental caries and tooth loss due to caries among adults is from cross-sectional studies, and surprisingly few population-level cross-sectional studies of dental caries among adults have been conducted. Only a small number of population-based cohort studies have followed adults through the third and fourth decades of life [Marmot and Brunner, 2005], and not one of these has, as yet, included the collection of dental data.

Studying the natural history of dental caries is important, as it will identify those periods in life when risk for disease is highest, and will indicate when and where intervention is most required (and allow analyses to estimate the benefit that an appropriate intervention may have on the population in question). A comprehensive picture of the natural history of caries experience in a population requires: (1) that the examined sample be representative (clinical or convenience samples are inappropriate because of their lack of generalizability); (2) that all permanent teeth be considered in the analysis; (3) separate observations for missing teeth (whether extracted due to caries or missing for some other reason) and for restorations or lesions in specific surfaces of teeth that are present in the mouth; and (4) that the prospective cohort study design be used.

Over recent years, several longitudinal studies of dental caries have been undertaken among older people 
[Chalmers et al., 2002a, b, 2005; Gilbert et al., 1999, 2001; Guivante-Nabet et al., 1999; Hamasha et al., 2005; Paulander et al., 2004; Thomson et al., 2002; Warren et al., 2002] and among children [Grindefjord et al., 1995, 1996; Karjalainen et al., 2001; Rodrigues and Sheiham, 2000; Thibodeau and O'Sullivan, 1999; Wendt et al., 1996, 1999]. However, there is a lack of sound published longitudinal data on adults in the middle years of life. Perhaps the closest has been a 10-year longitudinal study of a convenience sample of 587 persons aged 20-80 (440 followed up) from the People's Republic of China. In that study, 100 individuals aged 20-29 were examined at baseline (in 1984) and 78 were reexamined 10 years later; a further 100 individuals aged 30-39 were examined at baseline and 94 were reexamined at the 10-year followup. Only tooth-level DMFT (decayed, missing and filled teeth) data were reported. Among those aged 20-29 at baseline, the mean DMFT rose from 4.3 (SD 3.0) to 6.5 (SD 4.5) 10 years later. Among those aged 30-39 at baseline, the mean DMFT rose from 7.6 (SD 5.6) to 11.3 (SD 7.4) at follow-up [Luan et al., 2000]. It is difficult to generalize the findings of this study, however, as it used a convenience sample from a rural village in the People's Republic of China with little access to dental care, and the reported DMFT scores were aggregated for 10-year age groups with a 10-year follow-up.

There has also been a small 3-year radiographic study of caries experience in interproximal surfaces in 23-yearolds ( $n=69$ ) [Poorterman et al., 2003a; and a 6-year study in 17-year-olds: Poorterman et al., 2003b]; however, this was a radiographic study only (based on interproximal surfaces), and used a small convenience sample; thus, it is difficult to generalize from its findings.

It has been reported that it is difficult to draw comparisons between longitudinal and cross-sectional studies of dental caries experience; differing results may be obtained when cross-sectional and longitudinal analyses are applied to the same (longitudinal) data [Swedberg et al., 1997]. At any rate, estimates of caries experience and tooth loss from surveys have found that substantial caries experience has already occurred by the middle years of life, although its severity and extent appear to be decreasing in recent years. The US NHANES I (1971-1974) study reported a mean of 42.1 decayed, missing and filled surfaces (DMFS) in those aged 26-35 years, while the NHANES III (1988-1994) study reported a mean of just 25.7 DMFS in that age group [Brown et al., 2002]. Similar estimates were reported from NHANES III, Phase 1 (1988-1991), with a mean DMFS of 23.6 surfaces among dentate adults aged 25-34 years. A more recent cross-sec- tional study (November 2002 to March 2003) reported a mean DMFS of 9.6 (95\% CI 7.6, 11.6) among 116 Australian Army recruits aged between 26 and 30 [Hopcraft and Morgan, 2005]; however, estimates from this group are unlikely to be generalizable to the general population, as healthy, fit males are overrepresented in such a sample.

As can be seen from these studies, there is a paucity of recent information on dental caries and tooth loss experience among populations of adults in their twenties and thirties, and almost none on changes in disease experience among the same individuals over time. Accordingly, the aim of the current study is to describe in detail the natural history of dental caries and caries-related tooth loss in a population of young adults as they enter their middle years of life.

\section{Methods}

The Dunedin Multidisciplinary Health and Development Study (DMHDS) is a longitudinal study of a birth cohort of children who were born at the Queen Mary Hospital, Dunedin, New Zealand between April 1, 1972 and March 31, 1973 [Silva and Stanton, 1996]. Perinatal data were obtained and the sample for the longitudinal study was defined at age 3 years. This initially comprised 1,037 children assessed within 1 month of their third birthdays and again at ages 5, 7, 9, 11, 13, 15, 18, 21, 26 and, most recently, at 32 (with a dental examination also conducted at age 12). Barriers to study members' participation were minimized by the unit assuming the costs of participation (such as travel, lost wages, child care). Over $90 \%$ of the cohort self-identify as being of New Zealand European origin.

The various assessments (e.g. oral health, mental health, cardiovascular health) are presented as standardized modules in counterbalanced order, and each is conducted by a different examiner who is kept blind to all study data. The Otago Research Ethics Committee granted ethics approval for each assessment phase. Study members gave informed consent before participating.

\section{Measurement of Dental Caries Experience}

Sound archival longitudinal series dental clinical examination data (at the surface level) are available from previous phases of the study, with the exceptions of ages $7,11,13$, and 21 (when dental data were not collected). The current analysis used data from the dental examinations conducted at ages 26 and 32. Dental examinations for caries and missing teeth at age 26 were conducted using calibrated dental examiners. Teeth were examined for caries and restorations, with four surfaces being considered for anterior teeth (canines and incisors): buccal, lingual, distal, and mesial; a fifth surface, occlusal, was included for posterior teeth (premolars and molars). Diagnosis of caries was visual and made with the aid of a dental mouth mirror. Teeth were not dried prior to examination, sickle probes were used to remove plaque only, and radiographs were not taken. Where a surface could not be visualized by the examiner (due to excessive calculus or being covered by gingival tissue) the surface was excluded from the examination and later analyses. 
An estimate of accumulated tooth loss due to caries was obtained by observing the presence or absence of each tooth at age 26, and ascertaining the reason for its absence at that age by asking the study member at the time of the examination. In this study, only those teeth which had been lost because of caries were included in the analysis, and only teeth which had been lost because of caries are included in estimations of tooth loss due to caries and in the 'M' component of DMF scores. Third molar teeth were included in the analyses, as were crowned surfaces.

At age 32, dental examinations for caries and missing teeth were conducted by two calibrated examiners who examined approximately $50 \%$ of the dentally assessed study members each. Before examination of each study member, forms were adjusted to account for teeth that had been missing at the age 26 assessments. Protocols for the age 32 dental examinations mirrored those used at age 26. Replicate dental examinations were not possible during the assessments because of time constraints (due to the busy assessment day undergone by study members). However, protocols were followed strictly, and replicate examinations were conducted on a separate sample of 16 adults on four occasions during the Dunedin Study's age 32 data collection phase for assessment of examiner reliability. The intraclass correlation coefficient for intraexaminer reliability was 0.99 for both examiners and was also 0.99 for interexaminer reliability.

\section{Data Analysis}

Surface level dental examination data were used to compute DFS, $\mathrm{DM}_{3 \mathrm{~b}} \mathrm{FS}$, and $\mathrm{DM}_{5} \mathrm{FS}$ (generally accepted DMFS), and counts were also made of missing teeth (and whether these were missing due to caries or another reason). The $\mathrm{DM}_{3 \mathrm{~b}} \mathrm{FS}$ measure was described recently [Broadbent and Thomson, 2005], and involves assigning a minimum of three surfaces as being carious for an extracted tooth. Where more than three surfaces (four or five) are known to have been carious at the most recent assessment prior to the extraction of the tooth, this number of surfaces is assigned as having been carious instead. Scores for teeth that were missing due to caries from age 26 were adjusted by their surface status at age 18 , while teeth that were missing due to caries only at age 32 were adjusted by their surface status from age 26 [Broadbent and Thomson, 2005].

Crude caries increment $(\mathrm{CCI})$ and net caries increment $(\mathrm{NCI})$ were calculated. The CCI is a method of identifying the caries experience that occurs between two assessments, and involves comparing the baseline and follow-up status of each surface (on a surface-by-surface basis) in order to arrive at an estimate of the person-level disease increment. The NCI is a common method used to compensate for the effect of 'examiner' and 'true' caries 'reversals' upon DMFS scores. At the individual level, the number of reversals (negative increments) is subtracted from the number of positive caries increments to give the NCI [Beck et al., 1995; Broadbent and Thomson, 2005].

$$
\mathrm{CCI}=\frac{\sum_{\mathrm{i}=1}^{\mathrm{n}}\left(\text { events where surface sound } \mathrm{it}_{0} \text { but } \mathrm{DMF}_{\mathrm{it}_{1}}\right)}{\mathrm{n}}
$$$$
\mathrm{NCI}=\frac{\sum_{\mathrm{i}=1}^{\mathrm{n}}\left(\text { events where surface } \mathrm{DMF}_{\mathrm{it}_{0}} \text { but sound } \mathrm{it}_{1}\right)}{\mathrm{n}}
$$

Caries and Tooth Loss in a Birth Cohort
Table 1. Numbers taking part in the assessments at each age by gender

\begin{tabular}{lllr}
\hline & Males & Females & \multicolumn{1}{l}{ All } \\
\hline At age 26 & & & \\
$\quad$ Total surviving & $525(51.5)$ & $494(48.5)$ & $1,019(98.3)$ \\
$\quad$ Total assessed & $499(50.9)$ & $481(49.1)$ & $980(96.2)$ \\
$\quad$ Dentally examined & $472(50.8)$ & $458(49.2)$ & $930(91.3)^{\mathrm{a}}$ \\
At age 32 & & & \\
$\quad$ Total surviving & $523(51.5)$ & $492(48.5)$ & $1,015(98.0)$ \\
$\quad$ Total assessed & $495(50.9)$ & $477(49.2)$ & $972(95.8)$ \\
$\quad$ Dentally examined & $476(51.1)$ & $456(48.9)$ & $932(91.8)^{\mathrm{a}}$ \\
At age 26 and age 32 & & & \\
$\quad$ Total assessed & $475(49.3)$ & $489(50.7)$ & $964(95.0)$ \\
$\quad$ Dentally examined & $459(50.9)$ & $442(49.1)$ & $901(88.8)^{\mathrm{a}}$ \\
\hline
\end{tabular}

Figures in parentheses represent percentage.

${ }^{a}$ Two edentulous study members excluded.

Data were analyzed using the SPSS version 13.0.1. As this study is purely descriptive of caries and tooth loss experience between age 26 and 32, statistical tests were not performed.

\section{Results}

At age 26, 1,019 (98.3\%) of the 1,037 original study members were alive, and 980 (96\% of the surviving cohort) participated in the age 26 assessments. At age 32, 1,015 of the study members were alive, and 972 (96\% of the surviving cohort) were assessed. Dental examination data from both ages 26 and 32 were available for 901 study members (note that this figure excludes two completely edentulous study members). Approximately equal numbers of males and females were examined at both age 26 and 32 (table 1).

The mean number of teeth present in study members' mouths at age 26 was 28.6 (SD 2.2), and this number had dropped to 28.0 (SD 2.6) by age 32. Correspondingly, the number of tooth surfaces present fell from a mean of 131.4 (SD 11.0) at age 26 to 128.7 (SD 12.7) at age 32. The mean number of sites that were not visible on examination (due to being covered by calculus or excessive gingival tissue) also fell from a mean 1.8 (3.6) at age 26 to a mean 0.9 (2.4) at age 32. The prevalence of caries rose from $94.9 \%(\mathrm{n}=855)$ at age 26 to $96.8 \%(\mathrm{n}=872)$ by age 32 . At age 26,571 study members $(63.4 \%)$ had one or more decayed surfaces present. By age 32 , only 470 study members $(52.2 \%)$ had one or more decayed surfaces present. Between the ages of 26 and 32, the mean 
decayed surfaces remained about the same, while the mean filled surfaces increased (table 2).

The number of individuals who had lost one or more teeth due to caries increased from 97 to 205 (10.8 to $22.8 \%$ ) between ages 26 and 32, and 159 individuals (17.6\%) experienced a new caries-related tooth loss event during that period. The mean number of teeth lost due to caries almost trebled between ages 26 and 32, from 0.20 teeth (SD 0.71) at 26 to 0.58 teeth (SD 1.57) by age 32, comprising a mean increase of 0.38 teeth (table 3 ). Most tooth loss experience due to caries was in the posterior teeth, particularly the first and second molar teeth (fig. 1).
Table 2. Summary data on caries-related measures for study members assessed at both ages 26 and 32

\begin{tabular}{|c|c|c|c|c|}
\hline $\begin{array}{l}\text { Caries } \\
\text { severity }\end{array}$ & Age 26 & Age 32 & $\begin{array}{l}\text { CCI from } \\
26 \text { to } 32\end{array}$ & $\begin{array}{l}\text { NCI from } \\
26 \text { to } 32\end{array}$ \\
\hline Mean DS & $2.5(3.8)$ & $2.3(4.6)$ & $1.5(3.1)^{\mathrm{a}}$ & $0.8(3.1)^{\mathrm{a}}$ \\
\hline Mean FS & $10.3(9.7)$ & $12.4(10.9)$ & $2.2(3.2)$ & $1.8(3.2)$ \\
\hline Mean DFS & $12.8(10.8)$ & $14.7(12.0)$ & $3.7(4.5)$ & $2.6(4.4)$ \\
\hline Mean $\mathrm{DM}_{3 \mathrm{~b}} \mathrm{FS}$ & $13.4(11.6)$ & $16.5(13.0)$ & $4.3(5.3)$ & $3.3(5.2)$ \\
\hline Mean $\mathrm{DM}_{5} \mathrm{FS}$ & $13.9(12.3)$ & $17.7(15.9)$ & $5.0(6.6)$ & $3.9(6.5)$ \\
\hline
\end{tabular}

Figures in parentheses represent SD.

a Includes increments of 'filled' to 'filled and decayed'.

Table 3. Summary data on tooth loss experience of study members from age 26 to 32

\begin{tabular}{lrrr}
\hline & Age 26 & Age 32 & Experience 26-32 \\
\hline Number with 1 or more teeth lost due to caries $^{\mathrm{a}}$ & $97(10.8)$ & $205(22.8)$ & $159(17.6)$ \\
Number with 1or more teeth lost not due to caries $^{\mathrm{a}}$ & $448(54.2)$ & $559(64.3)$ & $181(20.1)$ \\
Mean number of teeth lost due to caries $^{\mathrm{b}}$ & $0.20(0.71)$ & $0.58(1.57)$ & $0.38(1.17)$ \\
Mean number of teeth lost not due to caries $^{\mathrm{b}}$ & $1.84(2.20)$ & $2.24(2.25)$ & $0.40(0.94)$ \\
\hline
\end{tabular}

${ }^{\text {a }}$ Figures in parentheses represent percentage.

${ }^{\mathrm{b}}$ Figures in parentheses represent SD.

Fig. 1. Percent of study members losing teeth due to caries by tooth type between ages 26 and 32 .

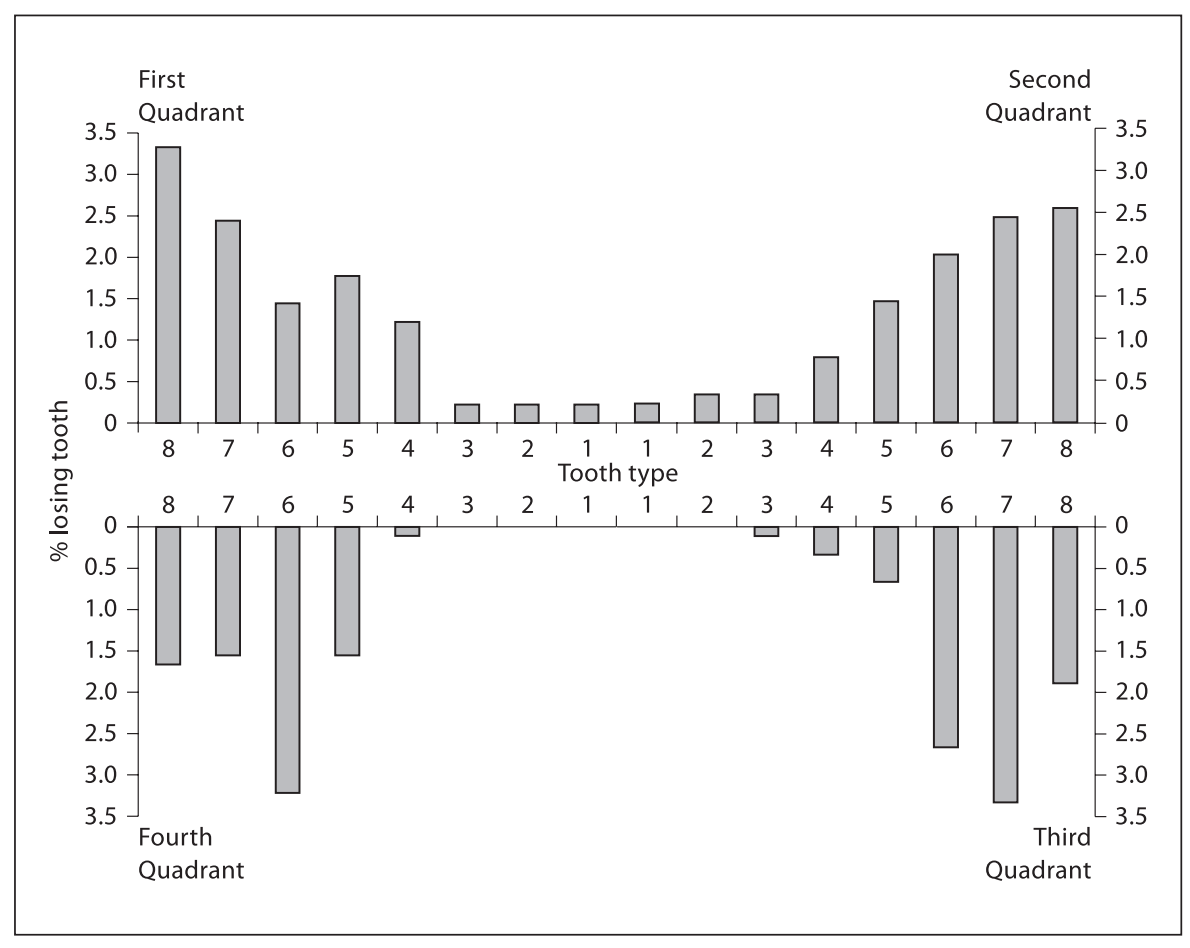


Tooth loss for reasons other than caries (including extractions of third molars and orthodontic extractions of premolars) also increased from age 26 to 32 , but to a lesser degree than for caries-related tooth loss (table 3 ).

Most of the caries experience between ages 26 and 32 occurred in the posterior teeth, particularly in the upper

Table 4. Tooth-level caries-related experience of study members between age 26 and age 32

\begin{tabular}{lcccc}
\hline \multicolumn{5}{l}{ Quadrants } \\
\cline { 2 - 5 } & first & second & third & fourth \\
\hline Third molar & $84(9.3)$ & $104(11.5)$ & $72(8.0)$ & $82(9.1)$ \\
Second molar & $193(21.4)$ & $169(18.8)$ & $161(17.9)$ & $174(19.3)$ \\
First molar & $138(15.3)$ & $140(15.5)$ & $182(20.2)$ & $180(20.0)$ \\
Second premolar & $108(12.0)$ & $116(12.9)$ & $89(9.5)$ & $97(10.8)$ \\
First premolar & $88(9.8)$ & $81(9.0)$ & $43(4.8)$ & $38(4.2)$ \\
Canine & $51(5.7)$ & $63(7.0)$ & $20(2.2)$ & $7(0.8)$ \\
Lateral incisor & $82(9.1)$ & $88(9.8)$ & $4(0.4)$ & $5(0.6)$ \\
Central incisor & $72(8.0)$ & $89(9.9)$ & $4(0.4)$ & $3(0.3)$ \\
\hline
\end{tabular}

Figures represent number of study members with increment, with the percentage given in parentheses. Transitions from sound to decayed, filled, filled and decayed, or missing, and unerupted to decayed, filled, filled and decayed, or missing are included. second premolars, upper molars, lower first molars, and lower second molars. Very little caries experience occurred in the anterior mandibular teeth, while a moderate amount occurred in the anterior maxillary teeth (table 4, fig. 2). Overall, the mean CCI was 5.0 surfaces (SD 6.6). Some $681(75.5 \%)$ experienced $1+\mathrm{CCI}$, and the mean CCI among these study members was 6.6 surfaces (SD 6.9).

At age 26, 17 study members had retained at least one deciduous tooth: 15 had one each, 2 had two each, and 1 had four. By age 32, the number with retained deciduous teeth had fallen to 14 . No dental implants were recorded at age 26; however, 6 study members had one or more implants at age 32 . Of those, 5 individuals had one implant each, and 1 had two implants. Finally, while no carious retained roots were observed at age 26, 21 individuals had one or more carious retained roots at age 32 (of whom 15 had one each, 5 had two each, and 1 had five).

\section{Discussion}

This is the first cohort study to report on changes in caries and tooth loss experience from the third to the fourth decade of life. The data show that substantial car-
Fig. 2. Mean number of caries events $\left(\mathrm{DM}_{3 \mathrm{~b}} \mathrm{FS}\right.$ crude caries increment) per study member by tooth type between ages 26 and 32.

Caries and Tooth Loss in a Birth Cohort

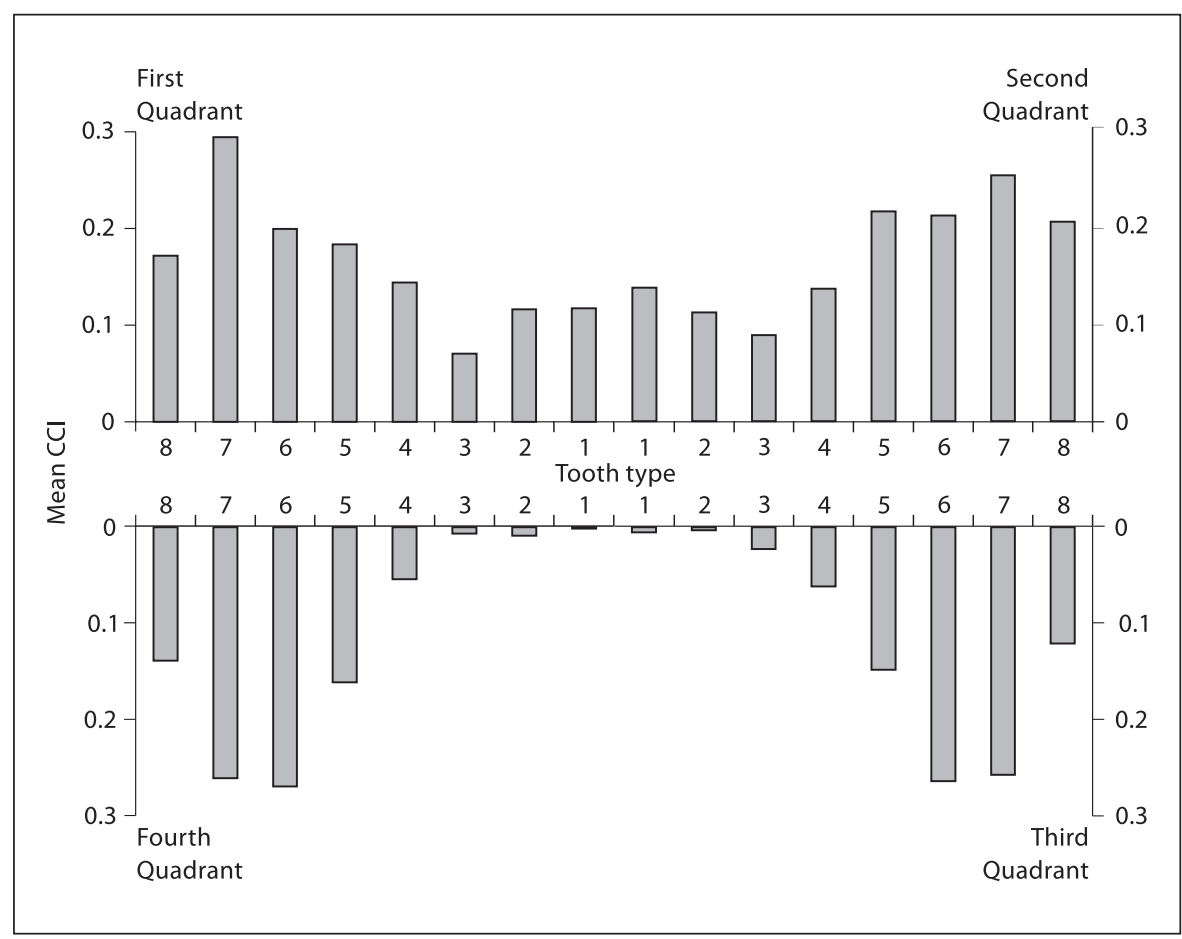

Caries Research 2006;40:459-465 
ies experience occurred during the 6-year period between the dental examinations. It is noteworthy that the annualized caries increment from this study is quite similar to estimates reported for adolescents and older people [Thomson, 2004]. The number of missing teeth and the number of restored surfaces increased substantially, while the number of decayed surfaces present in the mouth decreased slightly, possibly due to both ongoing incremental tooth loss and the placement of restorations.

Before discussing the study findings, it is appropriate to first consider the weaknesses and strengths of the study. Because teeth were not dried before the caries examination, and radiographs were not taken, it is likely that the data presented here are underestimates. It might also be argued that there are limitations to the applicability of New Zealand findings to other populations throughout the world; as with any other single-cohort longitudinal study, the Dunedin study produces knowledge which, inevitably, is bounded by time and place. On the other hand, the birth cohort is representative and of a sufficient size to support the relevance our estimates of prevalence to the general population. Furthermore, the Dunedin Study is the best-maintained longitudinal study in the world, with only $4 \%$ attrition over 32 years. Furthermore, the Dunedin Study is the only study to have followed changes in dental health from childhood to adulthood.

Where the study's strengths are concerned, the high retention rate and use of a complete birth cohort mean that the findings are generalizable to the source population. Furthermore, this is the first study to produce longitudinal population-level information on caries experience among adults entering their fourth decade of life. A problem with comparing the study findings to those of previous studies is the use of noncomparable age groups in previous studies (for example, broad 10-year age groups for follow-up comparisons in one longitudinal study [Luan et al., 2000]).

A large proportion of study members had experienced tooth decay between ages 26 and 32 . This increase in caries experience among the cohort was not surprising, given the increased exposure and time-at-risk of their tooth surfaces to dietary risk factors and bacterial plaque activity, as well as the failure of restorations [Axelsson et al., 2004; Harris et al., 2004]. That the increment rate for caries was greatest in the posterior and maxillary anterior teeth supports the findings of other studies [Macek et al., 2003].

By the age of 18 , only one of the study members who was also assessed at age 26 and 32 had lost $1+$ teeth due to caries; by age 26, 99 individuals had done so, and by age 32, 207 study members had. It is noteworthy that the mean incremental tooth loss due to caries between ages 26 and 32 (0.38 teeth) exceeded the entire mean life experience of tooth loss due to caries prior to age 26, which was a mean 0.20 teeth per person (table 3 ). This strongly suggests a markedly increased rate of caries-associated tooth loss occurring in the late twenties and early thirties.

In summary, substantial experience of dental restorations and tooth loss due to caries was observed among study members as they moved from the third decade of life into the fourth. The mean number of untreated carious surfaces showed a slight decrease, however. These findings may be generalized with a reasonable level of confidence to the population of New Zealand. Future oral health analyses with the Dunedin cohort will examine risk factors for dental caries and tooth loss, with particular attention to the association between socioeconomic status and oral health through the life course.

\section{Acknowledgments}

Jonathan Broadbent and the dental study are supported by Grant R01 DE-015260-01A1 from the National Institute of Dental and Craniofacial Research, National Institutes of Health, Bethesda, Md., USA. We thank study founder Phil Silva, Harvey Brown, all those involved in the administration of the study, and Air New Zealand. The Dunedin Study would not be possible but for the ongoing participation of the study members. The Health Research Council of New Zealand (previously the Medical Research Council), the New Zealand Department of Education, the New Zealand Department of Health, the National Children's Health Research Foundation, and the Dental Research Foundation of New Zealand provided funding for the assessments. 


\section{References}

Axelsson P, Nystrom B, Lindhe J: The long-term effect of a plaque control program on tooth mortality, caries and periodontal disease in adults. Results after 30 years of maintenance. J Clin Periodontol 2004;31:749-757.

Beck JD, Lawrence HP, Koch GG: A method for adjusting caries increments for reversals due to examiner misclassification. Community Dent Oral Epidemiol 1995;23:321-330.

Broadbent JM, Thomson WM: For debate: problems with the DMF index pertinent to dental caries data analysis. Community Dent Oral Epidemiol 2005;33:400-409.

Brown LJ, Wall TP, Lazar V: Trends in caries among adults 18 to 45 years old. J Am Dent Assoc 2002; 133:827-834.

Chalmers JM, Carter KD, Fuss JM, Spencer AJ, Hodge CP: Caries experience in existing and new nursing home residents in Adelaide, Australia. Gerodontology 2002a; 19:30-40.

- Chalmers JM, Carter KD, Spencer AJ: Caries incidence and increments in community-living older adults with and without dementia. Gerodontology 2002b;19:80-94.

Chalmers JM, Carter KD, Spencer AJ: Caries incidence and increments in Adelaide nursing home residents. Spec Care Dentist 2005;25: 96-105.

Gilbert GH, Duncan RP, Dolan TA, Foerster U: Twenty-four month incidence of root caries among a diverse group of adults. Caries Res 2001;35:366-375.

- Gilbert GH, Miller MK, Duncan RP, Ringelberg ML, Dolan TA, Foerster U: Tooth-specific and person-level predictors of 24-month tooth loss among older adults. Community Dent Oral Epidemiol 1999;27:372-385.

Grindefjord M, Dahllof G, Nilsson B, Modeer T: Prediction of dental caries development in 1year-old children. Caries Res 1995;29:343 348.

-Grindefjord M, Dahllof G, Nilsson B, Modeer T: Stepwise prediction of dental caries in children up to 3.5 years of age. Caries Res 1996;30:256266.
Guivante-Nabet C, Berenholc C, Berdal A: Caries activity and associated risk factors in elderly hospitalised population - 15-months follow-up in French institutions. Gerodontology 1999 16:47-58.

Hamasha AA, Warren JJ, Hand JS, Levy SM: Coronal and root caries in the older Iowans: 9- to 11-year incidence. Spec Care Dentist 2005;25: $106-110$.

Harris R, Nicoll AD, Adair PM, Pine CM: Risk factors for dental caries in young children: a systematic review of the literature. Community Dent Health 2004;21:71-85.

Hopcraft MS, Morgan MV: Comparison of radiographic and clinical diagnosis of approximal and occlusal dental caries in a young adult population. Community Dent Oral Epidemiol 2005;33:212-218.

Karjalainen S, Soderling E, Sewon L, Lapinleimu $\mathrm{H}$, Simell O: A prospective study on sucrose consumption, visible plaque and caries in children from 3 to 6 years of age. Community Dent Oral Epidemiol 2001;29:136-142.

Luan W, Baelum V, Fejerskov O, Chen X: Tenyear incidence of dental caries in adult and elderly Chinese. Caries Res 2000;34:205-213.

-Macek MD, Beltran-Aguilar ED, Lockwood SA, Malvitz DM: Updated comparison of the caries susceptibility of various morphological types of permanent teeth. J Public Health Dent 2003;63:174-182.

Marmot M, Brunner E: Cohort profile: the Whitehall II study. Int J Epidemiol 2005;34:251256.

Paulander J, Axelsson P, Lindhe J, Wennstrom J: Intra-oral pattern of tooth and periodontal bone loss between the age of 50 and 60 years. A longitudinal prospective study. Acta Odontol Scand 2004;62:214-222.

Poorterman JH, Aartman IH, Kieft JA, Kalsbeek $\mathrm{H}$ : Approximal caries increment: a three-year longitudinal radiographic study. Int Dent $\mathrm{J}$ 2003a;53:269-274.
Poorterman JH, Weerheijm KL, Aartman IH Kalsbeek H: Radiographic dentinal caries and its progression in occlusal surfaces in Dutch 17-year-olds: a 6-year longitudinal study. Caries Res 2003b;37:29-33.

Rodrigues CS, Sheiham A: The relationships between dietary guidelines, sugar intake and caries in primary teeth in low income Brazilian 3-year-olds: a longitudinal study. Int J Paediatr Dent 2000;10:47-55.

Silva PA, Stanton WR: From Child to Adult: the Dunedin Multidisciplinary Health and Development Study. London, Oxford University Press, 1996.

Swedberg Y, Freden H, Noren JG, Johnsson T: On longitudinal caries index data. A comparison study between cohort and cross-sectional attempts. Swed Dent J 1997;21:205-211.

Thibodeau EA, O’Sullivan DM: Salivary mutans streptococci and caries development in the primary and mixed dentitions of children. Community Dent Oral Epidemiol 1999;27:406412 .

Thomson WM: Dental caries experience in older people over time: what can the large cohort studies tell us? Br Dent J 2004;196:89-92.

Thomson WM, Spencer AJ, Slade GD, Chalmers $\mathrm{JM}$ : Is medication a risk factor for dental caries among older people? Community Dent Oral Epidemiol 2002;30:224-232.

-Warren JJ, Watkins CA, Cowen HJ, Hand JS, Levy SM, Kuthy RA: Tooth loss in the very old: 13 15 -year incidence among elderly Iowans. Community Dent Oral Epidemiol 2002;30:29-37.

Wendt LK, Hallonsten AL, Koch G: Oral health in pre-school children living in Sweden. III. A longitudinal study. Risk analyses based on caries prevalence at 3 years of age and immigrant status. Swed Dent J 1999;23:17-25.

Wendt LK, Hallonsten AL, Koch G, Birkhed D: Analysis of caries-related factors in infants and toddlers living in Sweden. Acta Odontol Scand 1996;54:131-137. 